\title{
YEAST PANEL Multiplex PCR Assay for Identification of Invasive Yeast Pathogens: Novel Diagnostic Strategy, Useful for Developing Countries
}

Amir Arastehfar ${ }^{1 *}$, Weniie Fang ${ }^{1,2,3^{*}}$, Weihua Pan ${ }^{2,3}$, Michaela Lackner ${ }^{7}$, Wanqing Liao ${ }^{2,3}$, Parisa Badiee $^{4}$, Kamiar Zomorodian $^{5}$, Hamid Badali $^{6}$, Ferry Hagen ${ }^{1}$, Cornelia Lass-Floerl $^{7}$, Teun Boekhout ${ }^{1,3,8}$ Westerdijk Fungal Biodiversity Institute, Utrecht, The Netherlands

Department of Dermatology, Shanghai Changzheng Hospital, Second Military Medical University, Shanghai, China

Shanghai Key Laboratory of Molecular Medical Mycology, Shanghai Institute of Mycology, Shanghai Changzheng Hospital, Second Military Medical University, Shanghai, China

Prof. Alborzi Clinical Microbiology Research Center, Shiraz University of Medical Sciences, Shiraz, Iran

Shiraz University of Medical sciences, Department of Parasitology and Mycology

Department of Medical Mycology and Parasitology, School of Medicine, Mazandaran University of Medical Sciences, Sari, Iran

Division of Hygiene and Medical Microbiology, Medical University of Innsbruck, Innsbruck, Austria

Institute of biodiversity and ecosystem dynamics, University of Amsterdam, Amsterdam, the Netherlands

\begin{abstract}
Introduction
The majority of invasive fungal infections (IFIs) are attributable to pathogenic yeast species Identification of pathogenic yeasts in developing countries are mainly performed by phenotypic and biochemical assays, which are time-consuming and inaccurate (1). This could lead to inaccurate epidemiological results and hence establishment of wrong empirical therapies, higher mortality rate, and emergence of resistant yeast pathogens (2). In order to solve this problem and due to the widespread use of PCR even in developing countries, a panel of three multiplex PCR assays (YEAST PANEL Multiplex PCR assay) targeting the most clinically important yeast pathogens were designed.
\end{abstract}

Materials and methods

Figure 1. Shows the validationn strategty utilzed for YEAST Panel multiplex PCR assay

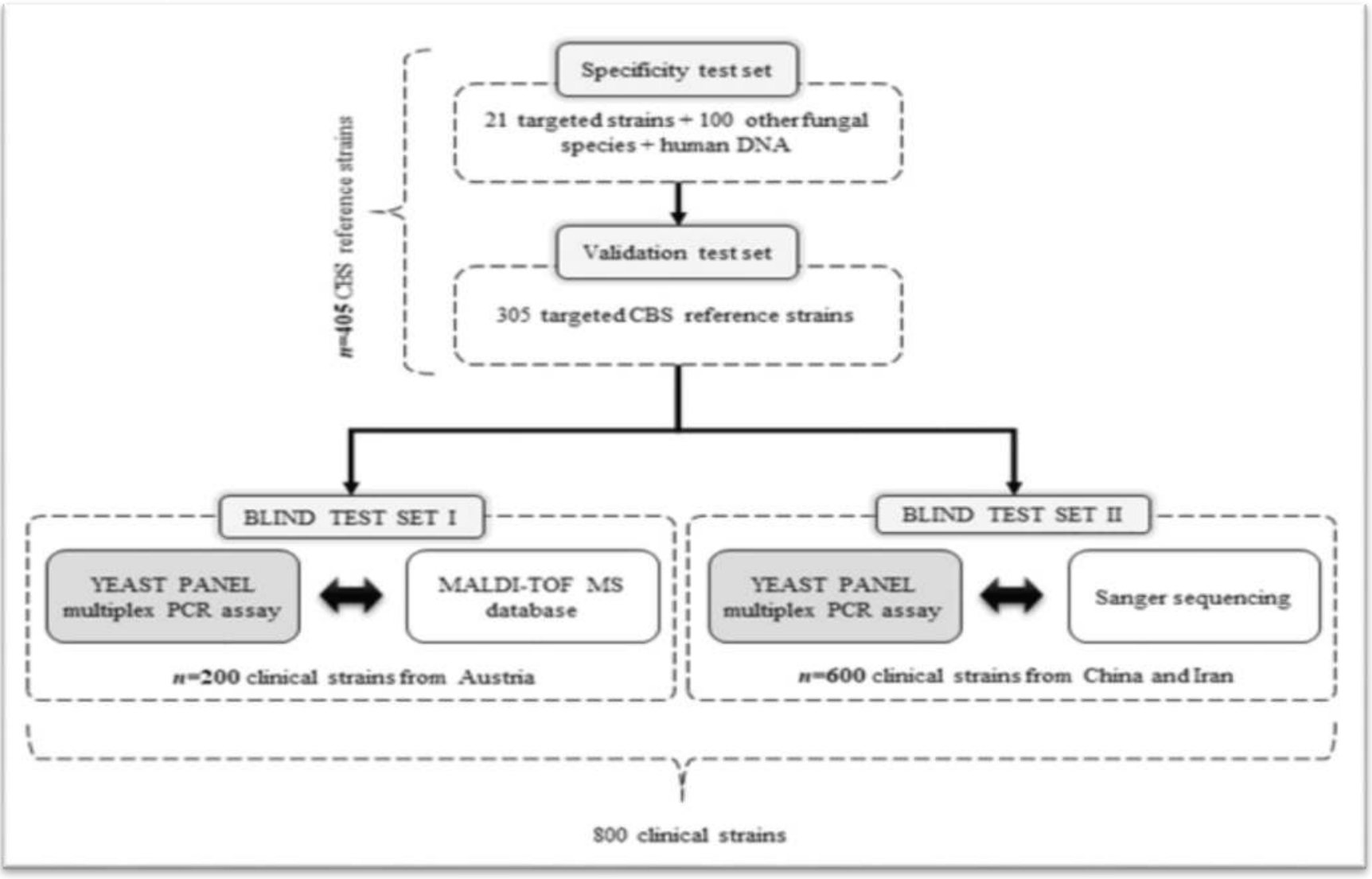

Results

Figure 2. A) Demonstrates the Step-wise strategy of YEAST Panel multiplex PCR

assay, and B) Illustrates the workflow of YEAST Panel multiplex PCR assay, starting

with yeast colonies, followed by PCR and running on the gel electrophoresis.
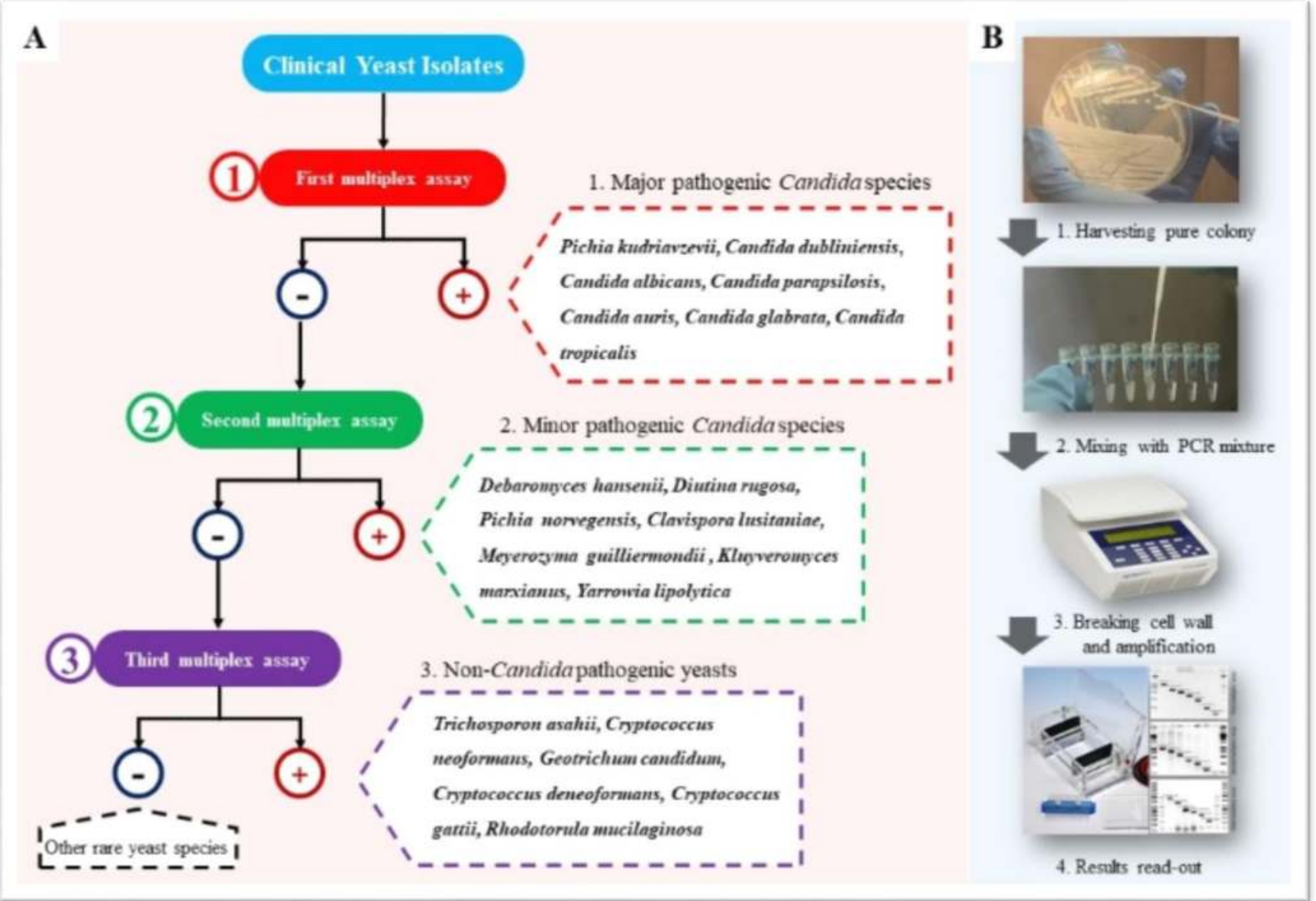

References

Kathuria S, Singh PK, Sharma C, Prakash A, Masih A, Kumar A, et al. Multidrug-resistant Candida auris misidentified as Candida haemulo 